\title{
Unconventional decay law for excited states in closed many-body systems
}

\author{
V.V. Flambaum ${ }^{1}{ }^{*}$ and F.M. Izrailev ${ }^{2}$ \\ 1 School of Physics, University of New South Wales, Sydney 2052, Australia \\ ${ }^{2}$ Instituto de Física, Universidad Autónoma de Puebla, Apartado Postal J-48, Puebla 72570, México
}

(October 31, 2018)

\begin{abstract}
We study the time evolution of an initially excited many-body state in a finite system of interacting Fermi-particles in the situation when the interaction gives rise to the "chaotic" structure of compound states. This situation is generic for highly excited many-particle states in quantum systems, such as heavy nuclei, complex atoms, quantum dots, spin systems, and quantum computers. For a strong interaction the leading term for the return probability $W(t)$ has the form $W(t) \simeq \exp \left(-\Delta_{E}^{2} t^{2}\right)$ with $\Delta_{E}^{2}$ as the variance of the strength function. The conventional exponential linear dependence $W(t)=C \exp (-\Gamma t)$ formally arises for a very large time. However, the prefactor $C$ turns out to be exponentially large, thus resulting in a strong difference from the conventional estimate for $W(t)$.
\end{abstract}

PACS numbers: 03.67.Lx, 05.45.Mt, 24.10.Cn

It is known that highly excited states can be treated as "chaotic" ones in many-body systems, such as complex atoms [1], multicharged ions [2], nuclei [3] and spin systems 4. 57. This happens due to a very high density of many-particle states, which strongly increases with an increase of energy. For example, in the case of $n$ Fermiparticles occupying the finite number $m$ of "orbitals" (single-particle states), the total number $N$ of many-body states grows exponentially fast with an increase of the number of particles, $N=m ! / n !(m-n) ! \sim \exp \left(c_{0} n\right)$. Correspondingly, the density $\rho_{f}$ of those many-body states which are directly coupled by a two-body interaction, also grows very fast [6]. Therefore, even a relatively weak interaction between the particles can lead to a strong mixing between unperturbed many-body states ("basis states"). As a result, an exact (perturbed) eigenstate is represented by a chaotic superposition of a large number of components of basis states.

The number of principal basis components in such chaotic eigenstates can be estimated as $N_{p c} \sim \Gamma / D$ where $\Gamma$ is the spreading width of a typical component, that can be estimated using the Fermi golden rule, and $D^{-1}(E)$ is the total density of many-body states. In the case of a quantum computer, the interval between multiqubit energy levels $D \propto 1 / N$ is extremely small, and practically it is impossible to resolve these levels. Moreover, both the temperature and finite time of computer operations lead to an energy uncertainty $\delta E \gg D$. A similar situation occurs for an electron which enters a many-electron quantum dot. In these cases the analysis of stationary chaotic eigenstates is not an adequate to real physical problems and one needs to consider the time evolution of wave functions. In this Letter we extend the quantum chaos approach to the problem of time evolution of an initially excited basis state.

Exact many-body eigenstates $|k\rangle$ of the Hamiltonian $H=H_{0}+V$ of interacting Fermi-particles can be expressed in terms of simple shell-model basis states $|f\rangle$ of $H_{0}$,

$$
|k\rangle=\sum_{f} C_{f}^{(k)}|f\rangle ; \quad|f\rangle=a_{f_{1}}^{+} \ldots a_{f_{n}}^{+}|0\rangle .
$$

Here $|0\rangle$ is the ground state, $a_{s}^{+}$is the creation operator and $C_{f}^{(k)}$ are components of an exact eigenstate in the unperturbed basis.

In application to quantum computer models the Hamiltonian $H_{0}$ describes a number of non-interacting qubits (two-level systems), and $V$ stands for the interqubit interaction needed for a quantum computation ( we assume time-independent $V$ ). In this case the basis state $\mid f>$ is a product of single qubit states, $a_{s}^{+}$is the spin-raising operator (if the ground state $|0\rangle$ corresponds to spins "down"), and chaotic eigenstates $|k\rangle$ are formed by the residual interaction $V$.

Below we consider the time evolution of the system, assuming that initially $(t=0)$ the system is in a specific basis state $|i\rangle$ (in the state with certain spins "up" for a quantum computer). This state can be expressed as a sum over exact eigenstates,

$$
|i\rangle=\sum_{k} C_{i}^{(k)}|k\rangle
$$

therefore, the time-dependent wave function reads as

$$
\Psi(t)=\sum_{k, f} C_{i}^{(k)} C_{f}^{(k)}|f\rangle \exp \left(-i E^{(k)} t\right) .
$$

*email address: flambaum@newt.phys.unsw.edu.au 
Here $E^{(k)}$ are the eigenvalues corresponding to the eigenstates $\mid k>$. The sum is taken over the eigenstates $\mid k>$ and basis states $\mid f>$ (in what follows, we put $\hbar=1$ ).

The probability $W_{i}=\left|A_{i}\right|^{2}=|\langle i \mid \Psi(t)\rangle|^{2}$ to find the system in the state $|i\rangle$ is determined by the amplitude

$$
\begin{array}{r}
A_{i}=\langle i|\exp (-i H t)| i\rangle=\sum_{k}\left|C_{i}^{(k)}\right|^{2} \exp \left(-i E^{(k)} t\right) \simeq \\
\int P_{i}(E) \exp (-i E t) d E .
\end{array}
$$

Here we replaced the summation over a large number of the eigenstates by the integration over their energies $E \equiv E^{(k)}$, and introduced the strength function (SF) $P_{i}(E)$ which is also known in the literature as the local spectral density of states,

$$
P_{i}(E) \equiv \overline{\left|C_{i}^{(k)}\right|^{2}} \rho(E) .
$$

Here $\rho(E)$ is the density of states of the total Hamiltonian $H$, and the average is performed over a number of states with close energies.

In chaotic systems the strength function $P_{i}(E)$ is known to have the Breit-Wigner form for a relatively weak interaction, and is close to the Gaussian for a strong interaction [1, 3. Recently, the following approximate general expression has been analytically found [7],

$$
\begin{aligned}
P_{i}(E)= & \frac{1}{2 \pi} \frac{\Gamma_{i}(E)}{\left(E_{i}+\delta_{i}-E\right)^{2}+\Gamma_{i}(E)^{2} / 4}, \\
& \Gamma_{i}(E) \simeq 2 \pi \overline{\left|V_{i f}\right|^{2}} \rho_{f}(E) .
\end{aligned}
$$

which is derived by making use of the approach described in Ref. [8]. Here $\Gamma_{i}(E)$ is some function of the total energy, $\delta_{i}$ is the correction to the unperturbed energy level $E_{i}$ due to the residual interaction $V_{i f}$, and $\rho_{f}(E)$ is the density of the basis states $|f\rangle$ directly connected with a given state $|i\rangle$ by the matrix element $V_{i f}$. The above result has been derived for the so-called Two-Body Random Interaction (TBRI) model [9] which describes $n$ interacting Fermi-particles distributed over $m$ orbitals, with an assumption that two-body matrix elements are completely random.

It is shown [7] that for a large number of particles the function $\Gamma_{i}(E)$ has the Gaussian form with the variance which depends on the model parameters. In the case of a relatively small (but non-perturbative) interaction (when $\Gamma_{i} \equiv 2 \pi \rho_{f} V_{0}^{2} \ll 2 \Delta_{E}$ with $\left.V_{0}^{2}=\left\langle V_{i f}^{2}\right\rangle\right)$, the function $\Gamma(E)$ is very broad (i.e. it does not change significantly within the energy intervals $\sim \Gamma$ and $\left.\Delta_{E}\right)$ and can be treated as constant, $\Gamma(E) \simeq \Gamma_{0}$. In the other limit case of a strong interaction, $\Gamma_{0} \geq \Delta_{E}$, the dependence $\Gamma(E)$ in (6) is the leading one.

The knowledge of the strength function allows one to describe the dynamics of wave packets in the energy space. It is easy to find the evolution of $W_{i}(t)$ on a small time scale. Let us subtract the energy $E_{i} \equiv H_{i i}$ of the initial state in the exponent and make a second order expansion in $E-E_{i}$ in Eq.(14). This gives the following result,

$$
A_{i}=\exp \left(-i E_{i} t\right)\left(1-\Delta_{E}^{2} t^{2} / 2\right)
$$

and

$$
W_{i}(t)=1-\Delta_{E}^{2} t^{2} .
$$

Here the width $\Delta_{E}$ of the SF is determined through the second moment, $\Delta_{E}^{2}=\sum_{f \neq i} V_{i f}^{2}$, which for the TBRI model is 10],

$$
\Delta_{E}^{2}=\frac{1}{4} V_{0}^{2} n(n-1)(m-n)(m-n+3),
$$

with $V_{0}^{2}$ standing for the variance of the off-diagonal elements $\left(\Delta_{E}^{2}\right.$ for a quantum computer model can be found in Ref. 11]).

Note that for a strong residual interaction, $\Gamma_{0} \geq \Delta_{E}$, the time dependence (9) is also correct for a longer time [11. Indeed, both the strength function and density of states in this limit are described by the Gaussian functions with the variance $\sigma^{2}=\Delta_{E}^{2}$ (see details in [9] - [13]),

$$
\begin{gathered}
P_{i}(E)=\frac{1}{\sqrt{2 \pi \sigma^{2}}} \exp \left[-\frac{\left(E-E_{c}\right)^{2}}{2 \sigma^{2}}\right], \\
\rho(E)=\frac{N}{\sqrt{2 \pi \sigma^{2}}} \exp \left(-\frac{E^{2}}{2 \sigma^{2}}\right) .
\end{gathered}
$$

Therefore, at the center of the energy spectrum, $E_{c}=0$, Eq.(14) results in the Gaussian time dependence for $A_{i}(t)$ and $W_{i}(t)$,

$$
\begin{gathered}
A_{i}=\exp \left(-\Delta_{E}^{2} t^{2} / 2\right), \\
W_{i}(t) \simeq \exp \left(-\Delta_{E}^{2} t^{2}\right) .
\end{gathered}
$$

Now let us consider large times. In this limit the result can be obtained by evaluation of the integral in Eq.(四) in the complex $E$-plane. Specifically, one should close the contour of integration in the bottom part of the complex plane (Im $E<0)$, in order to provide a vanishing contribution at infinity. Then, the large time limit is given by the pole of the strength function (6), closest to the real $E$-axis. If $\Gamma_{i}$ and $\delta_{i}$ in Eq.(5) do not depend on $E$, the integration gives the conventional exponential decay $W_{i}=\exp (-\Gamma t)[8]$. However, the energy dependence of $\Gamma$ is necessary to provide the finite second moment $\Delta_{E}^{2}$ of the strength function. If $\Gamma<\Delta_{E}$, the closest pole is given by $\tilde{\Gamma}=-2 \operatorname{Im} E_{p}$, where $E_{p}$ is the solution of the equation $E_{p}=E_{i}+\delta_{i}\left(E_{p}\right)-i \Gamma\left(E_{p}\right) / 2$ with a minimal 
imaginary part. If $\Gamma \ll \Delta_{E}$, then we have $\tilde{\Gamma} \approx \Gamma$. As a result, we obtain an exponential dependence for large $t$,

$$
W_{i}(t)=C \exp (-\tilde{\Gamma} t)
$$

with some constant $C$.

In Ref. 111 simple extrapolation formula for $W_{i}(t)$ has been suggested, that interpolates (for $\Gamma<\Delta_{E}$ ) between the small (9) and large (15) time dependencies,

$$
W_{i}(t)=\exp \left(\frac{\Gamma^{2}}{2 \Delta_{E}^{2}}-\sqrt{\frac{\Gamma^{4}}{4 \Delta_{E}^{4}}+\Gamma^{2} t^{2}}\right) .
$$

The transition between the Gaussian regime and simple exponential decay occurs near the time $t_{c} \sim \Gamma / \Delta_{E}^{2}$ where $\Delta_{E}^{2} t_{c}^{2} \sim \Gamma t_{c}$. This gives an estimate, $C \sim \exp \left(\frac{\Gamma^{2}}{2 \Delta_{E}^{2}}\right)$, for the constant $C$. Indeed, for $t<t_{c}$ the quadratic exponential decay, $\exp \left(-\Delta_{E}^{2} t^{2}\right)$, is slower than the linear one, $\exp (-\Gamma t)$. The matching of these two dependencies would naturally require the above expression for $C$. Thus, the constant $C$ can be large if $\Gamma>\Delta_{E}$. The transition from one regime of the time dependence of $W_{i}(t)$ to another is schematically shown in Fig.1.

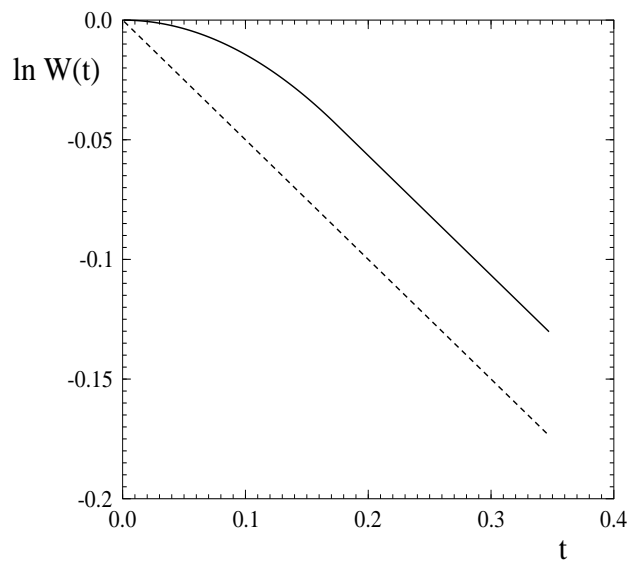

FIG. 1. Schematic time dependence $W(t)$ for $\tilde{\Gamma}=0.5$, $\Delta_{E}=1.2 ;$ the dependence $W(t)=\exp \left(-\Delta_{E}^{2} t^{2}\right)$ changes into $W(t)=\exp (-\Gamma t)$ at the point $t_{c}=\Gamma_{p} / \Delta_{E}^{2} \approx 0.17$. Simple exponential dependence is shown by the dotted line.

This qualitative result is supported by a more detailed consideration. In many-body systems the dependence $\Gamma(E)$ can be approximated by the Gaussian function since the density of final states $\rho_{f}\left(E_{f}\right)$ in Eq.(7) is typically close to the Gaussian [7]. This provides us with an approximate formula for the strength function (tested by the numerical calculations [14]),

$$
P(E)=B \frac{\exp \left[-\frac{\left(E-E_{0}\right)^{2}}{2 \sigma^{2}}\right]}{\left(E-E_{0}\right)^{2}+\Gamma^{2} / 4}
$$

It can be used, in conjunction with Eq.(4), to study the time dependence $W_{i}(t)$. Strictly speaking, this formula is valid near the center of the energy spectrum, otherwise one should take into account distortion effects near the edges of the energy spectrum.

Due to the normalization conditions, $\int P(E) d E=1$ and $\int E^{2} P(E) d E=\Delta_{E}^{2}$, we have the following relations |14,

$$
\frac{1}{B}=2\left[1-\Phi\left(\frac{\Gamma}{\sigma \sqrt{8}}\right)\right] \frac{\pi}{\Gamma} \exp \left(\frac{\Gamma^{2}}{8 \sigma^{2}}\right)
$$

and

$$
\Delta_{E}^{2}=B\left\{\sigma \sqrt{2 \pi}-\frac{\pi \Gamma}{2} \exp \left(\frac{\Gamma^{2}}{8 \sigma^{2}}\right)\left[1-\Phi\left(\frac{\Gamma}{\sigma \sqrt{8}}\right)\right]\right\}
$$

where $\Phi(z)$ is the error function.

The return probability $W(t)$ corresponding to the strength function (17) is defined by the integral,

$$
A(t)=B \int_{-\infty}^{\infty} d E \frac{\exp \left(-\frac{E^{2}}{2 \sigma^{2}}-i E t\right)}{\left(E-E_{0}\right)^{2}+\frac{\Gamma^{2}}{4}}
$$

The time dependencies of $A(t)$ and $W(t)$ for small time are given by Eqs.(13 14). If $\Gamma \ll \sigma$, the region of the applicability of these equations is very narrow. Indeed, in this case $\Delta_{E}^{2} \approx \frac{\sigma \Gamma}{\sqrt{2 \pi}}$ and the condition $t \ll t_{c} \ll \frac{\Gamma}{\Delta_{E}^{2}} \sim \frac{1}{\sigma}$ results in the relation $\Delta_{E}^{2} t^{2} \ll 1$. The absolute value of the amplitude $A(t)$ in this case is given by the series in the parameter $(\sigma t)^{2}=\left(t / t_{c}\right)^{2}$,

$$
|A(t)|=1-\frac{1}{2} \frac{\Gamma \sigma}{\sqrt{2 \pi}} t^{2}+\frac{1}{24} \frac{\Gamma \sigma^{3}}{\sqrt{2 \pi}} t^{4}+\ldots
$$

For large time, $t \gg t_{c}=1 / \sigma$, the calculation of the integral in Eq.(20) gives

$$
W(t) \approx \exp \left(\frac{1}{\pi} \frac{\Gamma^{2}}{\Delta_{E}^{2}}-\Gamma t\right)
$$

for the return probability. Here the correction $\frac{1}{\pi} \frac{\Gamma^{2}}{\Delta_{E}^{2}} \approx$ $\frac{2 \Gamma}{\sigma \sqrt{2 \pi}}$ is small. Indeed, the strength function in this case is close to the Lorentzian which gives a simple dependence $W(t)=\exp (-\Gamma t)$ for the decay.

Another limit case of a strong interaction, $\Gamma \gg \Delta_{E}$ (or, the same, $\Gamma \gg \sigma$ ), is more delicate. In this case the strength function is close to the Gaussian with $\Delta_{E} \approx \sigma$ and $t_{c}$ is large, $t_{c} \sim \frac{\Gamma}{\sigma^{2}} \gg \frac{1}{\sigma}$. The leading dependence of $W(t)$ in this case is the Gaussian, $W(t) \simeq \exp \left(-\Delta_{E}^{2} t^{2}\right)$ . Only for a long time $t \gg \frac{\Gamma}{\sigma^{2}}$ it becomes the simple exponential function,

$$
W(t) \approx \frac{\pi^{2} \Gamma^{2}}{8 \Delta_{E}^{2}} \exp \left(\frac{1}{4} \frac{\Gamma^{2}}{\Delta_{E}^{2}}-\Gamma t\right)
$$


It is important to stress that even for a large time the return probability $W(t)$ has large correction factor $\exp \left(\frac{1}{4} \frac{\Gamma^{2}}{(\Delta E)^{2}}\right)$, in addition to the standard decay law $\exp (-\Gamma t)$.

Due to a finite number of particles, there are specific important features in the dynamics of wave packets, namely, the damped oscillations and the break of the decay for $W_{i}(t)$ [15]. The number of basis components $|f\rangle$ within the energy shell $\left|E_{0}-E_{f}\right| \leq \min (\Gamma, \sigma) \equiv \Delta$ is finite. Therefore, the decay stops if $W_{i}$ is close to the equilibrium value defined as $W_{\infty} \equiv \overline{W_{i}(t \rightarrow \infty)} \approx 3 N_{p c}^{-1}$. Here $N_{p c}$ is the number of principal components in an eigenstate, $N_{p c} \sim \Delta / D$, where $D=\rho^{-1}$ is the mean energy interval between all many-body levels. Note that the value of $W_{\infty}$ is still at least 3 times larger than $W_{f}=N_{p c}^{-1}$ for any other component $f, \overline{W_{i}(\infty)} \geq$ $\overline{W_{f}(\infty)}$ (see, details in 15]).

The equilibrium occurs because the average decay flux is equal to the average return flux. However, the return flux leads to the damped oscillations of $W_{i}(t)$ and to the oscillations of a current number of the principal components $N_{p c}(t)$. These oscillations arise because the decay flux "reflects" from the edges of the energy shell, when all components within this shell are populated. Period of these oscillations is about $n_{c} / \Delta$ where $\Delta$ is the inverse decay time, and $n_{c}$ is the number of "classes" in the Hilbert space. This number can be defined as the number of interaction steps in the perturbative chain $H_{0 \alpha_{1}} H_{\alpha_{1} \alpha_{2}} \ldots H_{\alpha_{k} \alpha_{n_{c}}}$, needed to populate all basis states within the energy shell. For example, in the TBRI model with 6 particles and 12 orbitals, the number of steps is $n_{c}=3$ since each two-body interaction $H_{i k}$ moves only two particles to new orbitals.

In conclusion, we have studied generic features of the return probability $W(t)$ for a system to be found in an initially excited many-body state. Due to the two-body interaction between Fermi-particles, the wave packet in the energy representation spreads over all basis states within the energy shell. The dependence $W(t)$ for small time is determined by a ballistic spread of the packet and is given by the expression (9). For large time, the decrease of $W(t)$ is determined by the form of the strength function $P(E)$. We have analyzed the behavior of $W(t)$ by making use of the analytical expression for $P(E)$, which is obtained for any strength of random two-body interaction between finite number of interacting Fermi-particles.

We have shown that for the Breit-Wigner form of $P(E)$ (relatively weak interaction) the decay of $W(t)$ on a large time scale has the conventional exponential dependence, $W(t) \simeq \exp (-\Gamma t)$. On the other hand, for the Gaussian form of $P(E)$ (strong interaction) the time dependence $W(t)$ turns out to be of very specific. Namely, the leading term gives the quadratic exponential dependence, $W(t) \sim \exp \left(-\Delta_{E}^{2} t^{2}\right)$, and only for a very large time the conventional exponential linear dependence formally recovers. However, in this case an additional prefactor $C$ appears before the exponent, which turns out to be exponentially large, thus resulting in a strong modification of the standard exponential estimate for $W(t)$.

This work was supported by the Australian Research Council. One of us (F.M.I.) gratefully acknowledges the support by CONACyT (Mexico) Grant No. 34668-E. The authors are grateful to M.Yu. Kuchiev for valuable discussions.

[1] V. V. Flambaum, A. A. Gribakina, G. F. Gribakin, and M. G. Kozlov, Phys. Rev. A 50, 267 (1994).

[2] G.F. Gribakin, A.A. Gribakina, V.V. Flambaum. Aust. J.Phys. 52, 443 (1999).

[3] M.Horoi, V.Zelevinsky and B.A.Brown, Phys. Rev. Lett. 74, 5194 (1995); V.Zelevinsky, M.Horoi and B.A.Brown, Phys. Lett. B 350, 141 (1995); N.Frazier, B.A.Brown and V.Zelevinsky, Phys. Rev. C 54, 1665 (1996); V.Zelevinsky, B.A.Brown, M. Horoi and N.Frazier, Phys. Rep., 276 , 85 (1996).

[4] V. V. Flambaum, Proc. 85th Nobel Symposium, Phys. Scr. 46, 198 (1993).

[5] B. Georgeot and D. L. Shepelyansky, Phys. Rev. Lett. 81,5129 (1998).

[6] S. Aberg. Phys. Rev. Lett. 64, 3119 (1990). D.L.Shepelyansky and O.P.Sushkov, Europhys. Lett. 37, 121 (1997); B.L.Altshuler, Y.Gefen, A.Kamenev and L.S.Levitov, Phys. Rev. Lett., 78, 2803 (1997); A.D.Mirlin and Y.V.Fyodorov, Phys. Rev. B 56, 13393 (1997); D.Weinmann, J.-L. Pichard and Y.Imry, J.Phys. I France, 7, 1559 (1997); P.Jacquod and D.L.Shepelyansky, Phys. Rev. Lett. 79 , 1837 (1997); V.V.Flambaum and G.F.Gribakin, Phys. Rev. C 50, 3122 (1994); P.G.Silvestrov, Phys. Rev. Lett., 79, 3994 (1997); Phys. Rev. E. 58, 5629 (1998).

[7] V.V.Flambaum and F.M.Izrailev, Phys. Rev. E., 61, 2539 (2000).

[8] A. Bohr and B. Mottelson, Nuclear structure, Vol. 1 (Benjamin, New York, 1969).

[9] J. B. French and S. S. M. Wong, Phys. Lett. B 35, 5 (1970); O. Bohigas and J. Flores, Phys. Lett. B 34, 261 (1971).

[10] V.V.Flambaum and F.M.Izrailev, Phys. Rev. E 55, R13 (1997); E 56, 5144 (1997).

[11] V.V. Flambaum. Aust. J.Phys. 53, N4, (2000).

[12] T.A. Brody, J. Flores, J.B. French, P.A. Mello, A. Pandey, and S.S.M. Wong, Rev. Mod. Phys. 53, 385 (1981).

[13] V.K.B. Kota and R. Sahu, nucl-th/0006079.

[14] G.Casati, V.V.Flambaum, and F.M.Izrailev, to be published.

[15] V.V.Flambaum and F.M.Izrailev, to be published. 